\title{
Comparison of Spot Sign, Blend Sign and Black Hole Sign for Outcome Prediction in Patients with Intracerebral Hemorrhage
}

\author{
Peter B. Sporns, ${ }^{\mathrm{a}}$ Michael Schwake, ${ }^{\mathrm{b}}$ André Kemmling, ${ }^{\mathrm{c}}$ Jens Minnerup, ${ }^{\mathrm{d}}$ Wolfram Schwindt, ${ }^{\mathrm{a}}$ \\ Thomas Niederstadt, ${ }^{a}$ Rene Schmidt, ${ }^{e^{*}}$ Uta Hanning, ${ }^{\mathrm{a}, \mathrm{f}^{*}}$ \\ aDepartment of Clinical Radiology, University Hospital of Muenster, Muenster, Germany \\ ${ }^{b}$ Department of Neurosurgery, University Hospital of Muenster, Muenster, Germany \\ 'Institute of Neuroradiology, University Hospital of Luebeck, Luebeck, Germany \\ 'Department of Neurology, University Hospital of Muenster, Muenster, Germany \\ eInstitute of Biostatistics and Clinical Research, University of Muenster, Muenster, Germany \\ fDepartment of Diagnostic and Interventional Neuroradiology, University Hospital Hamburg-Eppendorf, Hamburg, Germany
}

Background and Purpose Blend sign (BS) and black hole sign (BHS) on non-contrast computed tomography (NCCT) and spot sign (SS) on CT-angiography (CTA) are indicators of early hematoma expansion in spontaneous intracerebral hemorrhage (ICH). However, their independent contributions to outcome have not been well explored.

Methods In this retrospective study, inclusion criteria were: 1) spontaneous ICH and 2) NCCT and CTA performed on admission within 6 hours after onset of symptoms. Discharge outcome was dichotomized as good (modified Rankin Scale [mRS] 0-3) and poor (mRS 4-6) outcomes. The impacts of BHS, BS and SS on outcome were assessed in univariate and multivariable logistic regression models.

Results Of 182 patients with spontaneous ICH, 26 (14.3\%) presented with BHS, 37 (20.3\%) with BS and $39(21.4 \%)$ with SS. There was a substantial correlation between SS and BS $(\kappa=0.701)$ and a moderate correlation between SS and BHS ( $\kappa=0.424)$. In univariable logistic regression, higher baseline hematoma volume $(P<0.001)$, intraventricular hemorrhage $(P=0.002)$ and the presence of $\mathrm{BHS} / \mathrm{BS} / \mathrm{SS}$ (all $P<0.001$ ) on admission CT scan were associated with poor outcome. Multivariable analysis identified intraventricular haemorrhage (odds ratio [OR] 2.22 per $\mathrm{mL}, P=0.022$ ), baseline hematoma volume (OR 1.03 per $\mathrm{mL}, P<0.001)$ and SS on CTA (OR 11.43, $P<0.001)$ as independent predictors of poor outcome, showing that SS compared to BS and BHS was more powerful to predict poor outcome.

Conclusions The NCCT BHS and BS are correlated with the CTA SS and are reliable predictors of poor outcome in patients with ICH. Of the CT variables indicating early hematoma expansion, SS on CTA was the most reliable outcome predictor. However, given their correlation with SS on CTA, BS and BHS on NCCT can be useful for predicting outcome if CTA is not obtainable.
Correspondence: Peter B. Sporns Department of Clinical Radiology, University Hospital of Muenster, Albert-Schweitzer-Campus 1 , Gebäude A1, 48149 Muenster, Germany

Tel: +49-251-84731

Fax: +49-251-8345064

E-mail: Peter.Sporns@ukmuenster.de

*Both senior authors contributed equally.

Received: December 22, 2016

Revised: June 7, 2017

Accepted: June 8, 2017

The authors have no financial conflicts of interest.

Keywords Cerebral hemorrhage; Computed tomography; Stroke; Hematoma 


\section{Introduction}

Early hematoma growth occurs in approximately one third of the patients with spontaneous intracerebral hemorrhage (ICH). ${ }^{1}$ In contrast to other predictors of poor neurological outcome, such as initial hematoma volume and location, hematoma growth is potentially modifiable if detected early. ${ }^{2-5}$

Recently different imaging characteristics for prediction of hematoma growth have been introduced recently ${ }^{6,7}$ including the black hole sign (BHS) obtainable in non-contrast computed tomography (NCCT). ${ }^{8}$ However, the impact of the BHS on neurological outcome and the association with more established imaging parameters such as the NCCT blend sign (BS) and spot sign (SS) visible in computed tomographic angiography (CTA) $)^{9-11}$ are not yet clear. In addition, it is unknown how the concurrent presence of each sign independently contributes to the predictive power of poor outcome.

The major advantage of the BHS and BS is the visibility in NCCT, which offers a greater availability in clinical routine. Additionally, allergic reactions to contrast medium and renal dysfunction present possible contraindications for contrast application. We therefore aimed to evaluate the association between NCCT BHS, NCCT BS and SS and their respective value for predicting clinical outcome.

\section{Methods}

\section{Patients}

We retrospectively studied our database for patients with $\mathrm{ICH}$ aged $\geq 18$ years between January 2010 and August 2015. As inclusion criteria we defined: 1) spontaneous ICH confirmed on NCCT and 2) NCCT and CTA performed on admission within 6 hours after onset of symptoms. Patients were excluded if they had head trauma, brain tumor or secondary ICH from hemorrhagic transformation of ischemic infarction. We defined a binary outcome (good vs. poor): as poor outcome we defined modified Rankin Scale (mRS) on discharge $>3$, good outcome was defined as $m R S \leq 3$. Additionally, we obtained vascular risk factors (hypertension, diabetes mellitus) from patients' clinical records.

The study was approved by the Ethics Committee of the University Muenster and the Westfalian Chamber of Physicians, Muenster, Germany. All study protocols and procedures were conducted in accordance with the Declaration of Helsinki.

\section{Imaging analysis}

The CT scans were performed using standard clinical parameters with axial 5-mm section thickness. The images were ob- tained and stored for further evaluation. The location of the hematoma was assessed and documented. Hemorrhage locations were classified as basal ganglia, lobe, brain stem and cerebellum. Two experienced readers independently first evaluated the presence of BHS and BS in all patients' NCCT and then independently evaluated the presence of SS in the corresponding CTA. Both readers were blinded to all clinical information and the other scans at the time of the ratings. Discrepancies about the occurrence of BHS, BS and SS were settled by joint discussion of the 2 readers.

The NCCT BHS was defined as recently published. ${ }^{8}$ Briefly, it consists of a relatively hypodense area (black hole) which is encapsulated within a hyperdense area and which is not connected with the adjacent brain tissue. The relatively hypodense area has an identifiable border and a difference of at least a 28 Hounsfield units (HU) between the 2 density regions. The BHS is illustrated in the Supplementary Figure 1.

The hematoma blend $\operatorname{sign}^{6}$ represents a hematoma with a hyperdense and a hypodense area, which show a well-defined margin that is easily recognized by the naked eye. There should be a difference of at least $18 \mathrm{HU}$ between the two regions and the hypodense area should not be encapsulated by the hyperdense part of the hematoma (Supplementary Figure 1).

The ICH volumes of the baseline CT were segmented using Analyze (Analyze 10.0; AnalyzeDirect Inc., Overland Park, KS, USA). We therefore extracted the Digital Imaging and Communications in Medicine (DICOM) data of CT scans and implemented them into Analyze. Here the hematoma volume was segmented slice by slice.

\section{Statistical analysis}

Univariable distribution of metric variables is described by median and interquartile range (IQR). Absolute and relative frequencies are given for categorical data. In order to compare two independent samples regarding a metric or categorical outcome we used Mann-Whitney U-test or Fisher's exact test, respectively. Mc Nemar's test was used to assess differences between paired portions.

Sensitivity, specificity, positive predictive value (PPV) and negative predictive value (NPV) of BHS, BS and SS are given with exact 95\% confidence interval (Cl). The underlying goldstandard was outcome (good or poor) as defined above in the patients section. We used McNemar's test to compare sensitivity or specificity of BHS and BS with that of SS, and we used the test of Leisenring et al. ${ }^{12}$ for analogous comparisons regarding PPV or NPV. In order to measure inter-rater agreement we used Cohen's kappa. ${ }^{13}$

Association between the clinical and radiological parameters 
and outcome (good or poor) was assessed by logistic regression analysis. For multivariable model building, stepwise forward selection was used with two-fold interactions being assessed in a second block (inclusion criterion: $P$-value of the score test $\leq 0.05$, exclusion criterion: $P$-value of the likelihood ratio test $>0.1$ ). Variables considered for multivariable model building are given in Table 1. Given for selected variables are odds ratio (OR) with $95 \% \mathrm{Cl}$ and $P$-value of likelihood ratio test. For non-selected variables $P$-value of score test is displayed. Odds were calculated as ratio of the probability for poor outcome to the probability for good outcome. Degree of agreement between imaging parameters was quantified using Cohen's kappa and visualized by Venn diagram.

No adjustment for multiple testing was performed. Accordingly, $P$-values are displayed for descriptive reasons in order to detect and study meaningful effects. The $P$-values are considered as noticeable in case of $P \leq 0.05$.

Statistical analyses were performed in SPSS version 24 (IBM

Table 1. Univariable analysis of predictors of poor outcome

\begin{tabular}{lccc}
\hline Baseline characteristics & OR & $95 \% \mathrm{Cl}$ & 0.202 \\
\hline Age at admission (years) & 1.012 & $0.993-1.032$ & 0.168 \\
Gender (ref: female) & 0.66 & $0.36-1.11$ & 0.062 \\
Bleeding localization & & & $0.486^{*}$ \\
$\quad$ Lobe vs. basal ganglia & 0.80 & $0.43-1.49$ & $0.026^{*}$ \\
$\quad$ Infratentorial vs. basal ganglia & 0.24 & $0.07-0.84$ & 0.183 \\
Hypertension & 1.49 & $0.83-2.70$ & 0.793 \\
Diabetes & 0.89 & $0.36-2.17$ & 0.002 \\
Intraventricular hemorrhage & 2.58 & $1.41-4.71$ & $<0.001$ \\
Intracerebral hemorrhage volume $(\mathrm{mL})$ & 1.04 & $1.02-1.06$ & $<0.001$ \\
Black hole sign & 11.40 & $2.61-49.89$ & $<.001$ \\
Blend sign & 8.56 & $2.90-25.51$ & $<.001$ \\
Spot sign & 13.24 & $3.90-44.95$ & $<$ \\
\hline
\end{tabular}

Univariable analysis of predictors of poor outcome using logistic regression. Given are $\mathrm{OR}$ with $95 \% \mathrm{Cl}$ and $P$-value of likelihood ratio test if not otherwise specified.

$\mathrm{OR}$, odds ratio; $\mathrm{Cl}$, confidence interval.

${ }^{*} P$-value of Wald test.

Table 2. Comparison of baseline demographic, clinical and radiological characteristics between patients with good outcome and those with poor outcome

\begin{tabular}{|c|c|c|c|c|}
\hline Baseline clinical and imaging characteristics & All $(n=182)$ & $\begin{array}{l}\text { Good outcome } \\
(m R S \leq 3)(n=78)\end{array}$ & $\begin{array}{l}\text { Poor outcome } \\
(m R S>3)(n=104)\end{array}$ & $P$ \\
\hline Age at admission (years) & $68(54-79)$ & $68(52-78)$ & $69(55-79)$ & 0.392 \\
\hline Female & $83(45.6)$ & $31(39.7)$ & $52(50.0)$ & 0.179 \\
\hline Hypertension & $99(54.4)$ & $38(48.7)$ & $61(58.7)$ & 0.229 \\
\hline Diabetes mellitus & $22(12.1)$ & $10(12.8)$ & $12(11.5)$ & 0.821 \\
\hline Bleeding location & & & & 0.108 \\
\hline Basal ganglia & $87(47.8)$ & $33(42.3)$ & $54(51.9)$ & \\
\hline Lobe & $81(44.5)$ & $35(44.9)$ & $46(44.2)$ & \\
\hline Brainstem & $6(3.3)$ & $5(6.4)$ & $1(1.0)$ & \\
\hline Cerebellum & $8(4.4)$ & $5(6.4)$ & $3(2.9)$ & \\
\hline Hematoma volume $(\mathrm{mL})$ & $22.4(8.3-42.7)$ & $11.1(4.7-26.6)$ & $31.5(55.3-79.0)$ & $<0.001$ \\
\hline Intraventricular hemorrhage & $101(55.5)$ & $33(42.3)$ & $68(65.4)$ & 0.003 \\
\hline Black hole sign & $26(14.3)$ & $2(2.6)$ & 24 (23.1) & $<0.001$ \\
\hline Blend sign & 37 (20.3) & $4(5.1)$ & 33 (31.7) & $<0.001$ \\
\hline Spot sign & 39 (21.4) & $3(3.8)$ & 36 (34.6) & $<0.001$ \\
\hline
\end{tabular}

Values are presented as median (interquartile range) or number (\%).

mRS, modified Rankin Scale. 
Corp., Armonk, NY, USA) and in SAS 9.4 (SAS Institute, Cary, NC, USA).

\section{Results}

\section{Baseline characteristics}

A total of 182 patients fulfilled the inclusion criteria, and were included in this analysis. The median age of the patients was 68 years (IQR 54-79). Eighty three (45.6\%) were female and 99
(54.4\%) were male. Poor outcome was observed in 104 patients (57.1\%). The median hematoma volume was $22.4 \mathrm{~mL}$ (IQR 8.342.7) and differed noticeably between patients with poor (median $31.5 \mathrm{~mL}$ ) and good outcome (median $11.1 \mathrm{~mL})(P<0.001)$. The hematoma was mostly located in the basalganglia $(n=87$, $47.8 \%)$, followed by cerebral lobes ( $n=81,44.5 \%)$, cerebellum $(n=8,4.4 \%)$ and brain stem $(n=6,3.3 \%)$. Analyses revealed statistically noticeable differences in the distribution of intraventricular hemorrhage $(P=0.003)$, hematoma volume $(P<0.001)$

Table 3. Sensitivity, specificity, PPV and NPV of BHS and BS as compared to SS

\begin{tabular}{|c|c|c|c|c|c|}
\hline & SS & BHS & $P($ BHS vs. SS) & BS & $P(\mathrm{BS}$ vs. SS) \\
\hline \multicolumn{6}{|c|}{ Any location $(n=182)$} \\
\hline Sensitivity & 34.6 (25.6-44.58) & $23.1(15.4-32.4)$ & 0.029 & $31.7(23.0-41.6)$ & 0.607 \\
\hline Specificity & 96.2 (89.2-99.2) & 97.4 (91.4-99.7) & 1.000 & 94.9 (87.4-98.6) & 1.000 \\
\hline PPV & $92.3(79.1-98.4)$ & $92.3(74.9-99.1)$ & 1.000 & $89.2(74.6-97.0)$ & 0.393 \\
\hline NPV & $52.4(43.9-60.9)$ & $48.7(40.7-56.8)$ & 0.563 & $51.0(42.6-59.4)$ & 0.347 \\
\hline \multicolumn{6}{|c|}{ Basal ganglia $(n=87)$} \\
\hline Sensitivity & $25.9(15.0-39.7)$ & $22.2(12.0-35.6)$ & 0.754 & $22.2(12.0-35.6)$ & 0.687 \\
\hline Specificity & 97.0 (84.2-99.9) & 97.0 (84.2-99.9) & 1.000 & 97.0 (84.2-99.9) & 1.000 \\
\hline PPV & 93.3 (68.1-99.8) & 92.3 (64.0-99.8) & 0.857 & 92.3 (64.0-99.8) & 0.901 \\
\hline NPV & $44.4(32.7-56.6)$ & $43.2(31.8-55.3)$ & 1.000 & $43.2(31.8-55.3)$ & 0.520 \\
\hline \multicolumn{6}{|l|}{ Lobe $(n=81)$} \\
\hline Sensitivity & $45.7(30.9-61.0)$ & $23.9(12.6-38.8)$ & 0.013 & 43.5 (28.9-58.9) & 1.000 \\
\hline Specificity & 94.3 (80.8-99.3) & 97.1 (85.1-99.9) & 1.000 & $91.4(77.0-98.2)$ & 1.000 \\
\hline PPV & $91.3(72.0-98.9)$ & 91.7 (61.5-99.8) & 0.970 & $87.0(66.4-97.2)$ & 0.293 \\
\hline NPV & $56.9(43.2-69.8)$ & $49.3(37.0-61.6)$ & 0.476 & $55.2(41.5-68.3)$ & 0.499 \\
\hline
\end{tabular}

Sensitivity, specificity, PPV, NPV of Spot Sign, Blend Sign and Black Hole Sign method to predict poor outcome. Values are presented as number (95\% confidene interval). $P$-values refer to pairwise comparison of BHS or BS with SS, respectively, and denote either $P$-value of McNemar's test (for sensitivity and specificity) or $P$-value of the test of Leisenring"1 (for PPV and NPV). For infratentorial location $(n=14)$ no measures are given due to small sample size. PPV, positive predictive value; NPV, vegative predictive value; BHS, black hole sign; BS, blend sign; SS, spot sign.

Table 4. Multivariable analysis of predictors for poor outcome: the final model

\begin{tabular}{lccc}
\hline Baseline characteristics & OR & $95 \% \mathrm{Cl}$ & $\mathrm{N} / \mathrm{S}: 0.597$ \\
\hline Age at admission (years) & - & - & $\mathrm{N} / \mathrm{S}: 0.097$ \\
Gender (ref: female) & - & - & $\mathrm{N} / \mathrm{S}: 0.270$ \\
Bleeding localization (ref: basal ganglia) & - & - & $\mathrm{N} / \mathrm{S}: 0.061$ \\
Hypertension & - & - & $\mathrm{N} / \mathrm{S}: 0.750$ \\
Diabetes & - & $1.12-4.44$ & 0.022 \\
Intraventricular hemorrhage & 2.22 & $1.01-1.05$ & $<0.001$ \\
Intracerebral hemorrhage volume $(\mathrm{mL})$ & 1.03 & - & $\mathrm{N} / \mathrm{S}: 0.116$ \\
Black hole sign & - & - & $\mathrm{N} / \mathrm{S}: 0.128$ \\
Blend sign & - & $3.25-40.24$ & $<0.001$ \\
Spot sign & 11.43 & - & \\
\hline
\end{tabular}

Multivariable analysis of predictors of poor outcome using stepwise forward selection in logistic regression (for details, see Methods). Given are OR with 95\% $\mathrm{Cl}$ and $P$-value of likelihood ratio test for selected variables. For non-selected variables $P$-value of score test is displayed. No interaction terms were selected. $\mathrm{OR}$, odds ratio; $\mathrm{Cl}$, confidence interval; $\mathrm{N} / \mathrm{S}$, not selected. 
and the imaging parameters SS, BS and BHS (all $P<0.001$ ) between patients with poor and good outcome (Table 2).

\section{Poor outcome of patients with BHS, BS and SS and interobserver agreement}

Of 182 patients with spontaneous ICH, 26 (14.3\%) presented with BHS, 37 (20.3 \%) with BS and 39 (21.4\%) with SS on initial imaging (Table 2). Fourteen (7.7 \%) patients had all of BHS, BS and SS, and all of them had a poor outcome. In contrast, of the remaining 168 patients, 68 (40.5\%) had a poor outcome. Inter-rater agreement for identifying BHS, BS and SS was very high between the 2 readers (BHS: $\kappa=0.934$, BS: $\kappa=0.965$, and SS: $\kappa=0.984)$. Discrepancy between the 2 readers was observed in 1 patient for SS, 3 for BHS, and 2 for BS.

The sensitivity, specificity, PPV, and NPV for predicting poor outcome were $23.1 \%, 97.4 \%, 92.3 \%$, and $48.7 \%$ for BHS, $31.7 \%, 94.9 \%, 89.2 \%$, and $51.0 \%$ for BS, and $34.6 \%, 96.2 \%$, $92.3 \%$, and $52.4 \%$ for SS (Table 3 ). SS showed a noticeably

Table 5. Agreement (Cohen's kappa) between SS, BS, and BHS

\begin{tabular}{lccc} 
& SS & BS & BHS \\
\hline SS & 1.000 & 0.701 & 0.424 \\
BS & 0.701 & 1.000 & 0.409 \\
BHS & 0.424 & 0.409 & 1.000
\end{tabular}

Cohen's kappa between SS, BS and BHS.

SS, spot sign; BS, blend sign; BHS, black hole sign.

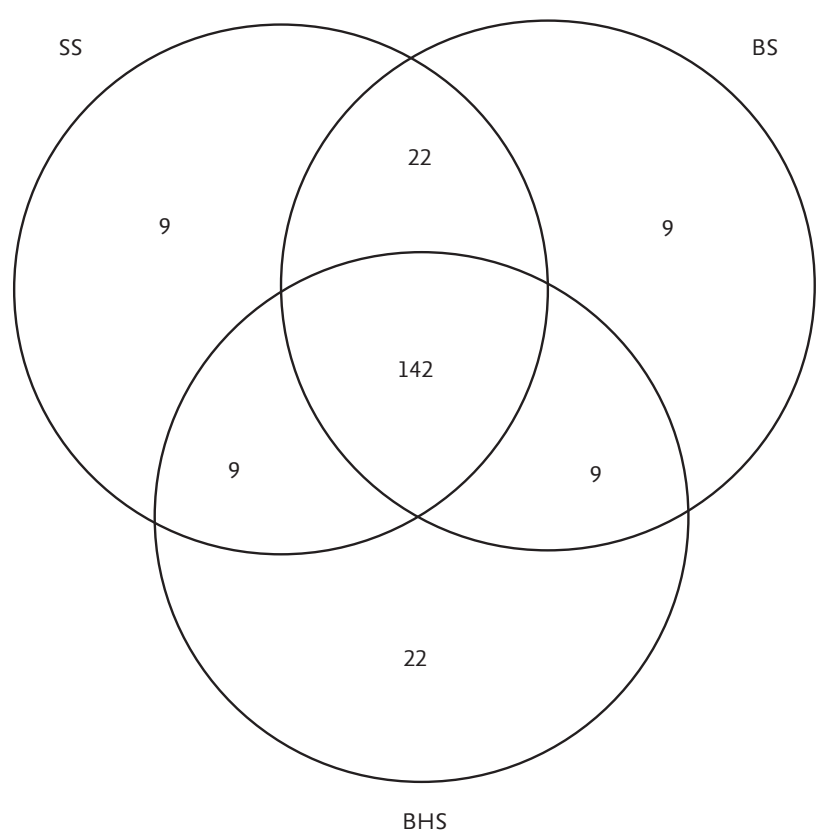

Figure 1. Venn diagram showing degree of agreement between imaging parameters blend sign (BS), black hole sign (BHS), and spot sign (SS). higher sensitivity for predicting outcome than BHS in any location (34.6\% vs. $23.1 \% ; P=0.029)$ and in the lobe ( $45.7 \%$ vs. 23.9\%; $P=0.013)$. Sensitivity of SS and BS were of comparable magnitude in any location ( $34.6 \%$ vs. $31.7 \% ; P=0.607$ ) and in the lobe (45.7\% vs. $43.5 \%$; $P=1.000)$. However, differences between SS vs. BHS and SS vs. BS regarding specificity, PPV and NPV were not statistically noticeable (Table 3 ).

Logistic regression analysis was performed to assess the association between various clinical and radiological parameters and poor outcome. In univariable logistic regression, higher baseline hematoma volume $(P<0.001)$, intraventricular hemorrhage $(P=0.002)$ and the presence of $\mathrm{BHS} / \mathrm{BS} / \mathrm{SS}$ (all $P<0.001)$ on admission CT scan were associated with poor outcome (Table 1).

Multivariable logistic regression analysis identified intraventricular hemorrhage (OR 2.22, $P=0.002$ ), higher baseline hematoma volume (OR 1.03 per $\mathrm{mL}, P<0.001$ ) and the presence of SS on baseline CT (OR 13.68, $P<0.001$ ) as independent predictors of poor outcome (Table 4). With these variables included in the model, presence of BHS and/or BS on baseline CT were not selected as further independent predictors for outcome in multivariable analysis $(P=0.116$ and $P=0.128)$. This reflects the high degree of association between imaging parameters that was substantial between BS and SS $(\kappa=0.701)$ and moderate between BHS and SS ( $\kappa=0.424)$ (Table 5). Outcome predictions were discrepant between BHS/BS and SS for only $31(17.0 \%) / 18$ $(9.8 \%)$ out of 182 patients (Figure 1). These results suggest good performance of BHS, BS and SS in prediction of poor outcome, with SS however remaining the strongest independent predictor of poor outcome compared to BS and BHS, and with

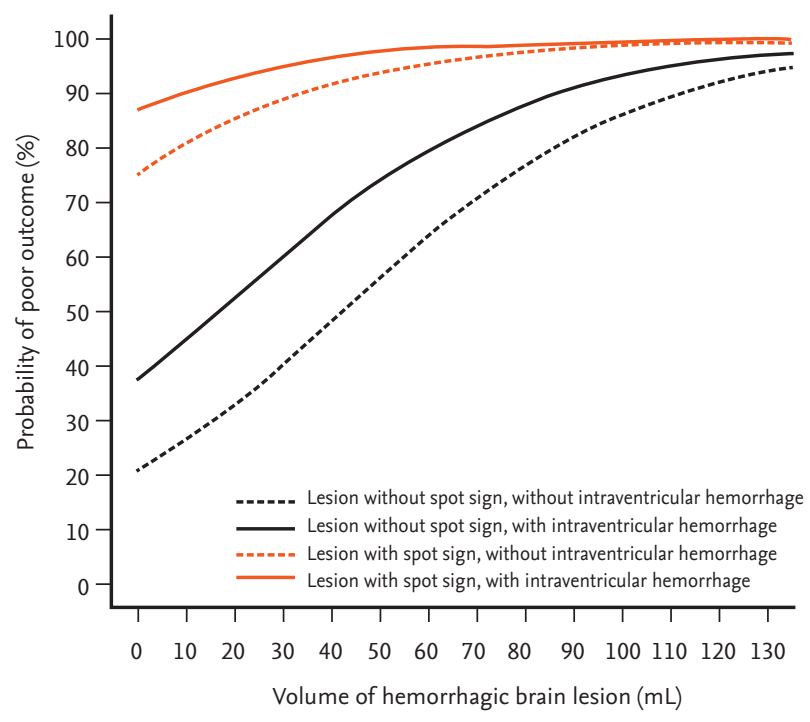

Figure 2. Predicted probability of poor outcome depending on ICH volume with and without spot sign, with and without intraventricular hemorrhage according to the final multivariable model (Table 4). 
BS performing slightly better than BHS.

According to the multivariable model, the probability of poor outcome is relevantly affected by the presence of SS (Figure 2). Whereas prognosis in patients without SS is poor only in the case of high hematoma volume, presence of SS is an indicator for poor outcome even for small hematoma volumes. For example, for a patient without intraventricular haemorrhage and hematoma volume of $20 \mathrm{~mL}$ the presence of SS increases the probability of poor outcome to $85 \%$, compared to $33 \%$ with absent SS, whereas for a patient without intraventricular haemorrhage and hematoma volume of $80 \mathrm{~mL}$ the presence of SS increases the probability of poor outcome to $97 \%$, compared to $76 \%$ with absent SS.

\section{Discussion}

Our results show that the NCCT imaging markers of BS and BHS are correlated with the established SS and are promising imaging parameters for prediction of poor neurological outcome. The prevalence of black hole sign and blend sign in our study was comparable to other studies. ${ }^{8}$ Even though the appearance of the black hole sign is less frequent compared to other signs, such as the blend sign, ${ }^{6}$ it offers a high specificity and PPV for prediction of poor neurological outcome and shows satisfying correlation with the presence of SS in the corresponding CTA.

Taking into account that SS has a higher sensitivity for outcome prediction than BHS or BS alone, both NCCT and CTA should be acquired if possible. Especially in a setting where CTA is not readily available or with strong contraindications for contrast application (distinct allergy, far progressed renal dysfunction), sole acquisition of noncontrast $\mathrm{CT}$ and evaluation of $\mathrm{BS}$ and BHS is a valuable option for detecting hematoma growth associated with poor outcome.

The high interrater reliability suggests that the BS and BHS are easy-to-use new imaging parameters. Good independent predictors of poor outcome also were baseline hematoma volume and intraventricular haemorrhage.

Limitations of our study are due to its retrospective nature and single center design. Another limitation is the missing long-term follow up that might offer additional information but were not available for this study. However, the large patient population suggests a high reliability and importance of these imaging parameters for outcome prediction in patients with ICH.

\section{Conclusions}

The NCCT BHS and BS are correlated with the CTA SS and are reliable predictors of poor outcome in patients with ICH. Of the CT variables indicating early hematoma expansion, SS on CTA was the most reliable outcome predictor. However, given their correlation with SS on CTA, BS and BHS on NCCT can be useful for predicting outcome if CTA is not obtainable.

\section{Supplementary materials}

Supplementary materials related to this article can be found online at https://doi.org/10.5853/jos.2016.02061.

\section{References}

1. Brott T, Broderick J, Kothari R, Barsan W, Tomsick T, Sauerbeck $L$, et al. Early hemorrhage growth in patients with intracerebral hemorrhage. Stroke 1997;28:1-5.

2. Davis $S M$, Broderick J, Hennerici M, Brun NC, Diringer MN, Mayer $\mathrm{SA}$, et al. Hematoma growth is a determinant of mortality and poor outcome after intracerebral hemorrhage. Neurology 2006;66:1175-1181.

3. Delcourt C, Huang Y, Arima H, Chalmers J, Davis SM, Heeley EL, et al. Hematoma growth and outcomes in intracerebral hemorrhage: the INTERACT1 study. Neurology 2012;79:314-319.

4. Fujii Y, Takeuchi S, Sasaki O, Minakawa T, Tanaka R. Multivariate analysis of predictors of hematoma enlargement in spontaneous intracerebral hemorrhage. Stroke 1998;29:11601166.

5. Broderick JP, Brott TG, Duldner JE, Tomsick T, Huster G. Volume of intracerebral hemorrhage. A powerful and easy-touse predictor of 30-day mortality. Stroke 1993;24:987-993.

6. Li Q, Zhang G, Huang YJ, Dong MX, Lv FJ, Wei X, et al. Blend sign on computed tomography: novel and reliable predictor for early hematoma growth in patients with intracerebral hemorrhage. Stroke 2015;46:2119-2123.

7. Blacquiere $D$, Demchuk AM, Al-Hazzaa M, Deshpande $A$, Petrcich $W_{1}$ Aviv RI, et al. Intracerebral hematoma morphologic appearance on noncontrast computed tomography predicts significant hematoma expansion. Stroke 2015;46:3111-3116.

8. Li $Q$, Zhang G, Xiong $X$, Wang XC, Yang WS, Li KW, et al. Black hole sign: novel imaging marker that predicts hematoma growth in patients with intracerebral hemorrhage. Stroke 2016;47:1777-1781.

9. Du FZ, Jiang R, Gu M, He C, Guan J. The accuracy of spot sign in predicting hematoma expansion after intracerebral hemorrhage: a systematic review and meta-analysis. PLoS One 
2014;9:e115777.

10. Del Giudice A, D'Amico D, Sobesky J, Wellwood I. Accuracy of the spot sign on computed tomography angiography as a predictor of haematoma enlargement after acute spontaneous intracerebral haemorrhage: a systematic review. Cerebrovasc Dis 2014;37:268-276.

11. Sporns PB, Schwake M, Schmidt R, Kemmling A, Minnerup J, Schwindt $W$, et al. Computed tomographic blend sign is as- sociated with computed tomographic angiography spot sign and predicts secondary neurological deterioration after intracerebral hemorrhage. Stroke 2017;48:131-135.

12. Leisenring $W_{1}$ Alonzo $T$, Pepe MS. Comparisons of predictive values of binary medical diagnostic tests for paired designs. Biometrics 2000;56:345-351.

13. Landis JR, Koch GG. The measurement of observer agreement for categorical data. Biometrics 1977;33:159-174. 

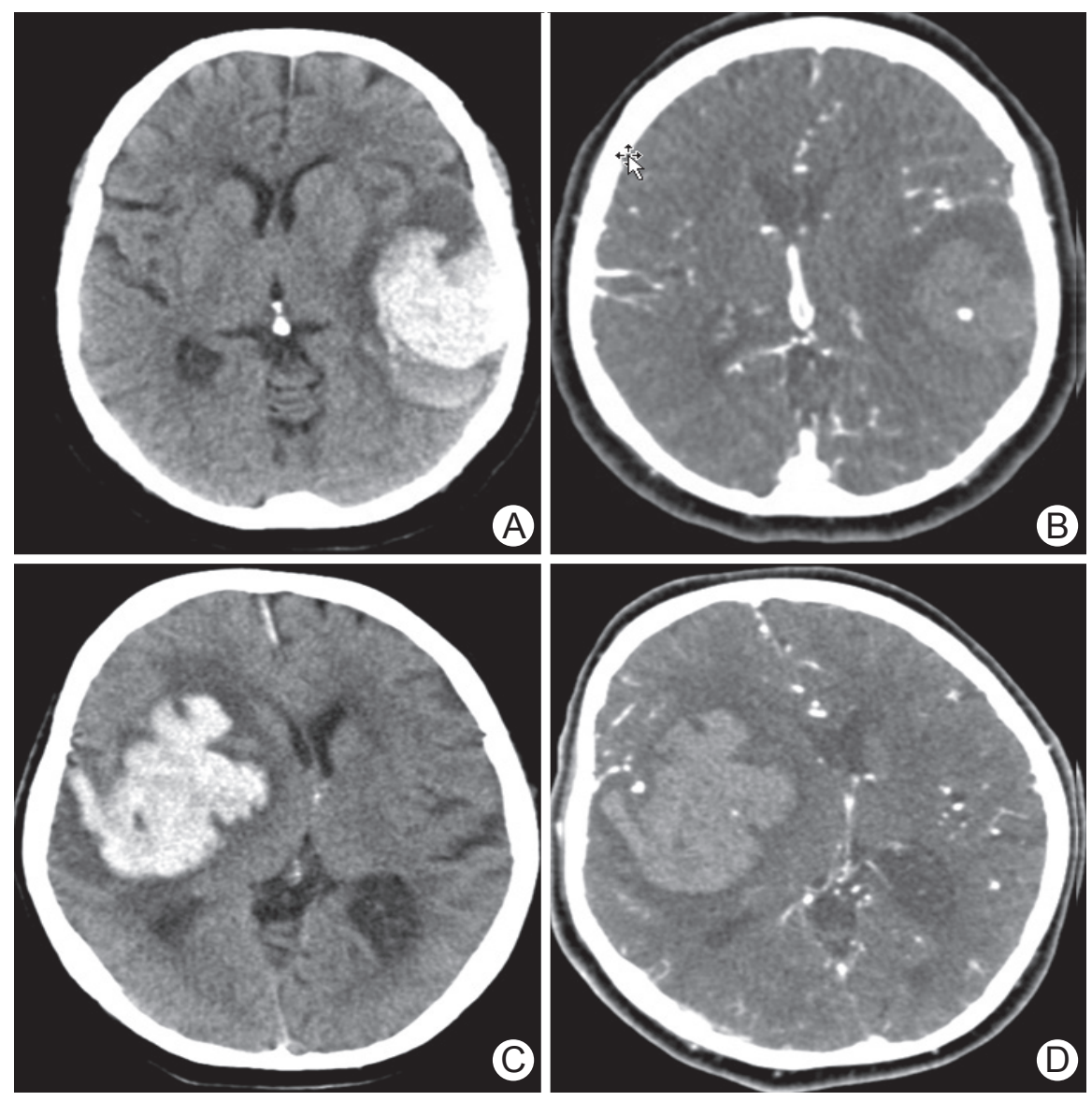

Supplementary Figure 1. (A) NCCT demonstrates blend sign with large cerebral hemorrhage in the left hemisphere. The hematoma consists of a hyperdense and a relatively hypodense part, which show a sharp margin. (B) In the corresponding CTA spot sign is visible within the hyperdense region of the hematoma. (C) The NCCT shows a black hole sign within the hyperdense hematoma. Note that the round BHS is not connected with the adjacent brain tissue. (D) Shows a spot sign in the corresponding CTA of the same patient. NCCT, non-contrast computed tomography; CTA, computed tomography angiography. 